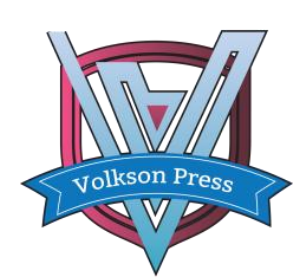

\author{
Contents List available at VOLKSON PRESS \\ Mechanical and Control Engineering (MCE) \\ DOI : http://doi.org/10.26480/wsmce.01.2017.60.64
}

\title{
BAND GAP CHARACTERISTICS OF ONE-DIMENSIONAL QUASI-PERIODIC PHONONIC CRYSTALS WITH LAYER THICKNESS ARRANGED BY TM (THUE-MORSE) SEQUENCE
}

\author{
Li Shang-da ${ }^{1,2}$, Liu Yan ${ }^{*}$ \\ ${ }^{1}$ China Ship Development and Design Center, Zhang Zhi-dong Road, Wuhan, China \\ ${ }^{2}$ Science and Technology on Ship Vibration and Noise Key Laboratory, Zhang Zhi-dong Road, Wuhan, China \\ *Corresponding Author email: liuyanhit@gmail.com
}

This is an open access article distributed under the Creative Commons Attribution License, which permits unrestricted use, distribution, and reproduction in any medium, provided the original work is properly cited

\section{ARTICLE DETAILS}

\section{Article History:}

Received 02 october 2017

Accepted 06 october 2017

Available online 11 november 2017

Keywords

phononic crystals, quasi-periodic, band structure, low frequency band gaps

\section{ABSTRACT}

In order to decrease the band gap frequency, a model of one dimensional quasi-periodic phononic crystals with layer thickness arranged by TM sequence was proposed. Its band structure was investigated numerically using the transfer matrix method combined with the supercell method, then its band gap characteristics were compared with those of periodic-structure phononic crystals. Besides, the effects of filling rate and layer-thickness rate were also discussed. The results show: the band gap splitting, and decreasing phenomena can be found in this type of quasiperiodic phononic crystals; its first band gap start frequency decline nearly $50 \%$, comparing to that in periodicstructure phononic crystals; when the rigid materials' filling rate and the elastic materials' approach parity, the band gap start frequency and stop frequency will come down; the first band-gap start frequency decrease with increment of layer-thickness rate. Arranging the geometrical parameters of periodic-structure phononic crystals in quasiperiodic sequence is one of the effective ways to declining the frequency of band gap, which benefits the practical application of phononic crystal isolators.

\begin{abstract}
1. Introduction
Phononic crystals are artificially periodic-structured acoustic-function composite materials, which provides excellent vibrational-waves bandpass and band-gap characteristics, so it has broad prospects in the controls of vibration and noise. Quasi-periodic phononic crystals are another functional composite material after the periodic-structure phononic crystals were put forward [1]. They are a kind of intermediate structure, which not only retains the basic physical properties of periodic structure system, but also shows specific physical characteristics due to the more abundant physical parameters, between the periodic and complete random structure.
\end{abstract}

In general, the application value of phononic-crystals band gap manifests itself in two ways: the band gap position and its width. The lower the band gap frequency and the wider the width, the higher the practical value is [2]. The giant band gaps can be obtained by uniting or nesting different band gaps of different phononic crystals, which will multiply the size of the phononic-crystals isolators and cause the difficulty of its application in the limited-space ship cabin. Besides, if the size of periodic-structure phononic crystals is under restrictions, the band gap frequency will be very high. It is unsuccessful that dealing with the vibration and noise problem caused by ship mechanical equipment in the middle and low frequency (below $500 \mathrm{~Hz}$ ) [3]. Therefore, the quasi-periodic property is introduced into the periodic structure to obtain the low frequency band gap, which becomes a research focus of phononic crystals [4].

A group of researchers calculated the transmission coefficient of the phononic crystal with material parameters arranged by Fibonacci sequence. They found that the strong localized resonance state is, comparing with the periodic structure, the most significant physical characteristics of quasi-periodic phononic crystals [5]. In addition, they also has studied the propagation characteristics of low order lamb waves in the one-dimensional thin composite plates with material parameters arranged by Fibonacci sequence. It is proved that the lamb-wave band gaps in this kind of quasi-periodic composite thin plate is split [6]. A group researcher studied the transmission characteristics of one dimensional piezoelectric phononic crystal with material parameters arranged by Fibonacci sequence. The results show that the band gaps of this structure are broadened, and the incident angle is an important factor affecting the band gap [7]. Xiao and the team investigated the propagation characteristics of elastic waves and the basic characteristics of local resonant modes in the solid/liquid phononic crystal with symmetric quasi-periodic structure, using the transfer matrix method [8]. Also in other studies, a group researcher also studied the properties of onedimensional rod phononic crystals with material parameters arranged by TM sequence using the finite element method. The results show that the splitting and decreasing phenomena of the band gaps in quasi-periodic phononic crystals can be found, and the band gap characteristics are independent of the generation of TM sequence [9].

The research of quasi-periodic phononic crystals mainly focus on quasiperiodic phononic crystals with material parameters arranged by quasi sequence itself and its band gap characteristics. The structure of this kind of quasi-periodic phononic crystals is relatively single. In the practical engineering, because of the limited material selection (cannot be adjusted and changed freely), obtaining the low frequency band gap by adjusting the materials has some limitations.

In allusion to these problems, this paper presents a kind of phononic crystals with geometrical parameters arranged by quasi-periodic sequence: one dimensional quasi-periodic phononic crystals with layer thickness arranged by TM sequence. In this paper, the transfer matrix method combined with the supercell method will be used to calculate the band structure, the effects of filling rate and layer-thickness rate on the band gap characteristics will be analyzed too. It can be a way to obtain the low frequency band gap, which provides a reference for the wide application of phononic crystals.

\section{MODAL OF ONE DIMENSIONAL QUASI-PERIODIC PHONONIC CRYSTALS WITH LAYER THICKNESS ARRANGED BY TM SEQUENCE}

There are many kinds of quasi-periodic sequences, such as Fibonacci sequence, Thue- Morse sequence (TM sequence), Period-Doubling sequence and their evolutions.

Compared with any other kinds of quasi-periodic sequences, the TM sequence only adjusts the elements' order, changes slightly, and does not change the elements' proportion in the periodic sequence. So, in the phononic-crystals isolators, TM sequence shows many advantages, such as not changing isolator's total height, total mass and average density. 
Therefore, this paper takes only the phononic crystal with two kinds of materials whose thickness arranged by TM sequence. The other cases are not repeat.

The TM sequence satisfies the following iteration series [10]:

$$
\left\{\begin{array}{l}
G_{1}=\mathrm{A} \\
G_{2}=\mathrm{AB} \\
G_{n}=G_{n-1} \overline{G_{n-1}} \quad(n \geq 2)
\end{array}\right.
$$

Where A and B are two elements that are arranged in TM sequence; $G_{n}$ is the n-generation TM sequence, $\overline{G_{n-1}}$ is the reverse of $G_{n}$.

From the iterative relationship described above, we can see that the total number of elements in $G_{n}$ is $2^{n-1}$, and the number of elements A and B are equal.

Assuming that the elements $\mathrm{A}$ and $\mathrm{B}$ are made of the materials 1 and 2 , the thickness of material 1 in $\mathrm{A}$ and $\mathrm{B}$ are $w_{1}+\Delta w_{1}, w_{1}-\Delta w_{1}$, the thickness of material 2 in $\mathrm{A}$ and $\mathrm{B}$ are $w_{2}+\Delta w_{2}, w_{2}-\Delta w_{2}$, and $\Delta w_{1}$, $\Delta w_{2}$ are not zero at the same time, the one dimensional quasi-periodic phononic crystals with layer thickness arranged by TM sequence can be formed, when A and B are arranged by TM quasi sequence along the $\mathrm{x}$ axis direction, as shown in fig. 1 below.

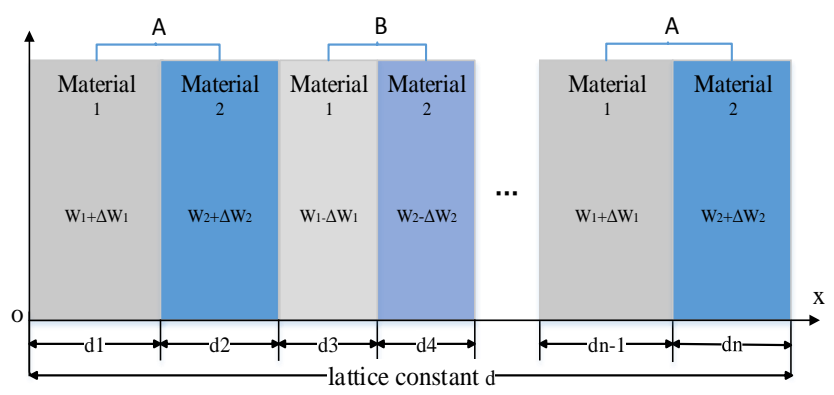

Figure 1: The Supercell of One Dimensional Quasi-Periodic Phononic Crystals with Layer Thicknesses Arranged by TM Sequence

$d$ is the lattice constant, $d_{1}, d_{2}, d_{3}, \ldots, d_{n}$ are the thickness of the materials 1 and 2, respectively, when A and B are arranged by TM quasi sequence. As shown in fig. 1, the geometric parameters (the layer thickness of materials 1 and 2) in this structure are arranged by the TM quasi sequence, but the material parameters are still arranged by the periodic sequence. Assuming that the supercell is the q-generation TM sequence, the total number of layers in the supercell is $n=2 \times 2^{q-1}$, including the number of layers of material 1 and that of the material 2 .

If we calculate the band structure of the supercell using the transfer matrix method, the generalized eigenvalue equation can be obtained by the displacement and stress continuity conditions of elastic waves at interfaces, and the Bloch theorem $[1,2,11,12]$ :

$$
|\mathbf{T}-\exp (i k d) \mathbf{I}|=0
$$

Where $\mathbf{T}$ is the transfer matrix, $\mathrm{k}$ is one dimensional scalar-form Bloch wave vector, $\mathbf{I}$ is the second order identity matrix? Solving the eigenvalue of the matrix $\mathbf{T}$, the dispersion relation between wave vector and frequency can be obtained.

\section{NUMERICAL CALCULATION AND ANALYSIS}

When composed of two kinds of materials, one dimensional quasi-periodic phononic crystals with layer thickness arranged by TM sequence consist of two types of situations: one is that only one of the material 1's thickness and the material 2's is arranged by TM sequence, which can be called single-change TM quasi-periodic phononic crystals; The other is that both of the material 1's thickness and the material 2's are arranged by TM sequence, which can be called double-changes TM quasi-periodic phononic crystals.
In this paper, the band structures of these two situations of the quasiperiodic phononic crystals with layer thickness arranged by TM sequence are calculated. The difference of the band gap from the periodic structure, and the effects of the filling rate and the layer-thickness rate on the band gaps of one dimensional quasi-periodic phononic crystals with layer thickness arranged by TM sequence are analyzed.

\subsection{Band Gap Characteristics of Single-Change TM Quasi-Periodic Phononic Crystals}

If the 4-generation TM sequence is the chosen one, taking changing layer thickness of the material 2 as an example, the band gap characteristics of the single-change TM quasi-periodic phononic crystals are analyzed.

The layer thickness of material 1 in $\mathrm{A}$ and $\mathrm{B}$ are $5 \mathrm{~mm}$, remaining unchanged. The layer thickness of material 2 in A and B are $7.5 \mathrm{~mm}, 2.5$ $\mathrm{mm}$, respectively. Besides, the material parameters are shown in table 1 below.

Table 1: Material Parameters

\begin{tabular}{ccccc}
\hline & Names & $\rho\left(\mathrm{kg} / \mathrm{m}^{3}\right.$ & $E\left(10^{10} \mathrm{~Pa}\right.$ & $\begin{array}{c}\text { Poisson } \\
\text { Ratio }\end{array}$ \\
\hline material & $\mathrm{Pb}$ (Plumbum) & 11600 & 4.08 & 0.369 \\
material & Silicone & 1300 & $1.175 \times 10.5$ & 0.464 \\
2 & Rubber & & & \\
\hline
\end{tabular}

Figure 2 is the band structure diagram of the single-change TM quasiperiodic phononic crystal.

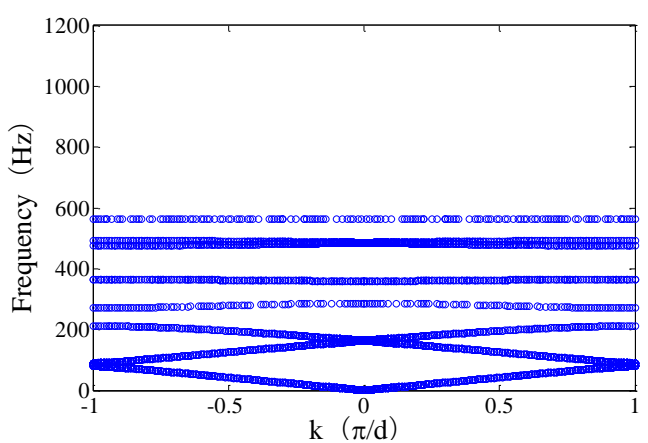

Figure 2: The Band Structure of the Single-Change TM Quasi-Periodic Phononic Crystal

From figure 2, the first band gap of the single-change TM quasi-periodic phononic crystal is $211.4 \sim 270.3 \mathrm{~Hz}$, the second band gap is $286.1 \sim 359.4 \mathrm{~Hz}$, the third band gap is $365.1 \sim 476 \mathrm{~Hz}$, the fourth band gap is $492.8 \sim 563.7 \mathrm{~Hz}$, and the start frequency of the fifth band gap is $564 \mathrm{~Hz}$.

For ease of comparison, a corresponding periodic structure phononic crystal is set, that is to say, the thickness of material 1 and 2 in the periodic structure phononic crystal are $w_{1}=5 \mathrm{~mm}, w_{2}=5 \mathrm{~mm}$, respectively. So $\Delta w_{1}=\Delta w_{2}=0$. Its band structure has been shown in the fig. 3 .

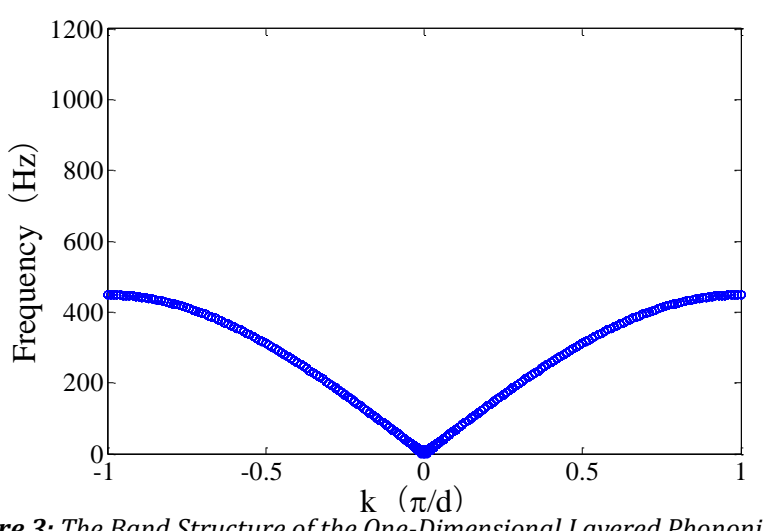

Figure 3: The Band Structure of the One-Dimensional Layered Phononic Crystal

As shown in figure 3, the start frequency of the first band gap of the onedimensional layered phononic crystal is $448.7 \mathrm{~Hz}$. So, if we change one dimensional layered phononic crystal into its corresponding one- 
dimensional quasi-periodic phononic crystal with layer thickness arranged by TM sequence, the start frequency of the first band gap will decreases by $237.3 \mathrm{~Hz}$, that is, cut about $53 \%$. Because of the periodic of the higher space in quasi-periodic phononic crystals, the band structure changed dramatically [13]. For example, in the frequency range of $210 \sim 1200 \mathrm{~Hz}$, there are four very narrow passbands, which split the band gap into five parts. Therefore, arranging the geometric parameters by quasi-periodic sequence is also an effective way to reduce the band-gap start frequency of the phononic crystals isolators, which is beneficial to its practical application.

\subsection{Band Gap Characteristics of Double-Changes TM Quasi-Periodic Phononic Crystals}

When the layer thickness of material 1 in $\mathrm{A}$ and $\mathrm{B}$ are set into $w_{1}+\Delta w_{1}=7.5 \mathrm{~mm}, w_{1}-\Delta w_{1}=2.5 \mathrm{~mm}$, respectively, and the thickness of the material 2 is consistent with that of the 3.1, the band structure of the double-changes TM quasi-periodic phononic crystal is shown in fig. 4 below.

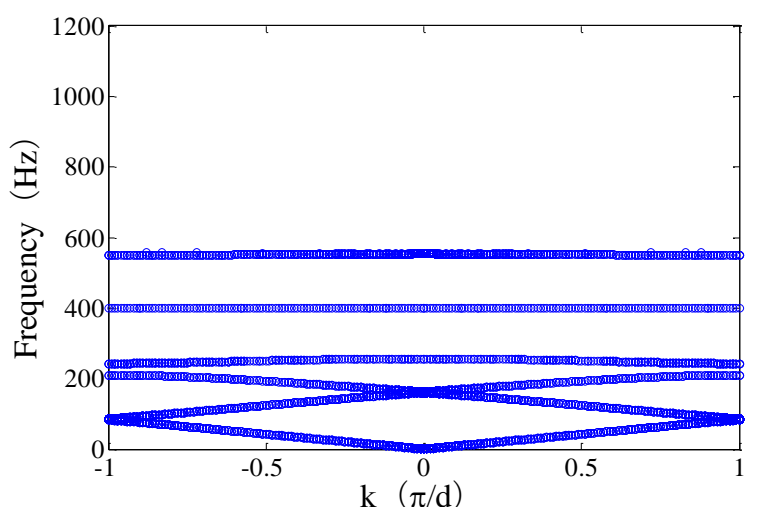

Figure 4: The Band Structure of the Double-Changes TM Quasi-Periodic Phononic Crystal

From figure 4, we can see that the first band gap of the double-changes TM quasi-periodic phononic crystal is $209.8 \sim 242.4 \mathrm{~Hz}$, the second band gap is $256.1 \sim 398.5 \mathrm{~Hz}$, the third band gap is $399.5 \sim 550.2 \mathrm{~Hz}$, and the start frequency of the fourth band gap is $557.2 \mathrm{~Hz}$.

Compared with the single-change TM quasi-periodic phononic crystal, the first 3 band gaps of the double-changes TM quasi-periodic phononic crystal are redistributed, and the total bandwidth increase. The start frequency of the first band gap changes slightly, although its bandwidth narrows. The width of the second and third band gaps increase, the start frequency of the second band gap moves to lower frequency. Due to the stop frequency of the first band gap, both of the third band-gap start frequency and stop frequency move to higher frequency. Thus, exchanging between the single-change TM quasi-periodic phononic crystal and the double-changes can be a useful means to adjust the distribution of band gaps. For all above, the structure of quasi-periodic phononic-crystals isolators should be selected according to the spectrum characteristics of the mechanical equipment, in engineering practice.

\subsection{Effects of Filling Rate on the Band Gaps}

The filling rate of material $1(\mathrm{~Pb})$ can be described as $\eta_{1}=w_{1} /\left(w_{1}+w_{2}\right)$, similarly, the filling rate of material 2 (Silicone Rubber) as $\eta_{2}=w_{2} /\left(w_{1}+w_{2}\right)=1-\eta_{1}$.

According to the dispersion relation of quasi-periodic phononic crystals with layer thickness arranged by TM sequence, the effects of filling rates $\eta_{1}$ and $\eta_{2}$ on the first 3 band gaps in the frequency range of $0 \sim 1200 \mathrm{~Hz}$ are studied, results are shown in table 2 4 below.

Table 2: The Effects of Filling Rate on the First Band Gap

\begin{tabular}{|c|c|c|c|c|c|c|}
\hline $\begin{array}{l}\text { Filling } \\
\text { Rates }\end{array}$ & Single-Change & $\begin{array}{l}\text { TM Quasi-Periodic } \\
\text { Crystals }\end{array}$ & Phononic & Double-Change & $\begin{array}{l}\text { TM Quasi-Periodi } \\
\text { Crystals }\end{array}$ & Phononic \\
\hline$\eta_{2}$ & $\begin{array}{l}\text { Start Frequency } \\
(\mathrm{Hz})\end{array}$ & $\begin{array}{c}\text { Stop Frequency } \\
(\mathrm{Hz})\end{array}$ & $\begin{array}{c}\text { Bandwidth } \\
(\mathrm{Hz})\end{array}$ & $\begin{array}{c}\text { Start Frequency } \\
(\mathrm{Hz})\end{array}$ & $\begin{array}{l}\text { Stop Frequency } \\
(\mathrm{Hz})\end{array}$ & $\begin{array}{c}\text { Bandwidth } \\
\text { (Hz) }\end{array}$ \\
\hline
\end{tabular}

\begin{tabular}{cc|ccc|ccc}
\hline $1 / 3$ & $2 / 3$ & 217.5 & 274.2 & 56.7 & 216.2 & 249.1 & 32.9 \\
$2 / 5$ & $3 / 5$ & 212.5 & 269.7 & 57.2 & 211.1 & 243.5 & 32.4 \\
$1 / 2$ & $1 / 2$ & 211.4 & 270.3 & 58.9 & 209.8 & 242.4 & 32.6 \\
$3 / 5$ & $2 / 5$ & 218.1 & 280.2 & 62.1 & 216.3 & 250 & 33.7 \\
$2 / 3$ & $1 / 3$ & 227.9 & 293.5 & 65.6 & 225.9 & 261.2 & 35.3 \\
\hline
\end{tabular}

Table 3: The Effects of Filling Rate on the Second Band Gap

\begin{tabular}{cc|ccc|ccc}
\hline \multicolumn{2}{c|}{$\begin{array}{c}\text { Filling } \\
\text { Rates }\end{array}$} & \multicolumn{4}{|c|}{ Single-Change TM Quasi-Periodic Phononic } \\
Crystals & \multicolumn{3}{|c}{ Double-Changes TM Quasi-Periodic Phononic } \\
Crystals & \\
$\eta_{1}$ & $\eta_{2}$ & $\begin{array}{c}\text { Start Frequency } \\
(\mathrm{Hz})\end{array}$ & $\begin{array}{c}\text { Stop Frequency } \\
(\mathrm{Hz})\end{array}$ & $\begin{array}{c}\text { Bandwidth } \\
(\mathrm{Hz})\end{array}$ & $\begin{array}{c}\text { Start Frequency } \\
(\mathrm{Hz})\end{array}$ & $\begin{array}{c}\text { Stop Frequency } \\
(\mathrm{Hz})\end{array}$ & $\begin{array}{c}\text { Bandwidth } \\
(\mathrm{Hz})\end{array}$ \\
\hline $1 / 3$ & $2 / 3$ & 293 & 367.5 & 74.5 & 265.2 & 401.5 & 136.3 \\
$2 / 5$ & $3 / 5$ & 286.9 & 360.1 & 73.2 & 258.3 & 397.5 & 139.2 \\
$1 / 2$ & $1 / 2$ & 286.1 & 359.4 & 73.3 & 256.1 & 398.5 & 142.4 \\
$3 / 5$ & $2 / 5$ & 295.6 & 371.4 & 75.8 & 263.5 & 414 & 150.5 \\
$2 / 3$ & $1 / 3$ & 309.1 & 388.4 & 79.3 & 275 & 434.1 & 159.1 \\
\hline
\end{tabular}

Table 4: The Effects of Filling Rate on the Third Band Gap

\begin{tabular}{cc|ccc|ccc}
\hline \multicolumn{2}{c|}{$\begin{array}{c}\text { Filling } \\
\text { Rates }\end{array}$} & \multicolumn{4}{|c|}{ Single-Change TM Quasi-Periodic Phononic } \\
Crystals & \multicolumn{3}{|c}{ Double-Changes TM Quasi-Periodic Phononic } \\
Crystals & & & \\
$\eta_{1}$ & $\eta_{2}$ & $\begin{array}{c}\text { Start Frequency } \\
(\mathrm{Hz})\end{array}$ & $\begin{array}{c}\text { Stop Frequency } \\
(\mathrm{Hz})\end{array}$ & $\begin{array}{c}\text { Bandwidth } \\
(\mathrm{Hz})\end{array}$ & $\begin{array}{c}\text { Start Frequency } \\
(\mathrm{Hz})\end{array}$ & $\begin{array}{c}\text { Stop Frequency } \\
(\mathrm{Hz})\end{array}$ & $\begin{array}{c}\text { Bandwidth } \\
(\mathrm{Hz})\end{array}$ \\
\hline $1 / 3$ & $2 / 3$ & 375 & 487.1 & 112.1 & 403.2 & 550.4 & 147.2 \\
$2 / 5$ & $3 / 5$ & 366.7 & 477.3 & 110.6 & 398 & 554 & 156 \\
$1 / 2$ & $1 / 2$ & 365.1 & 476 & 110.9 & 399.5 & 550.2 & 150.7 \\
$3 / 5$ & $2 / 5$ & 376.8 & 491.4 & 114.6 & 415 & 572.6 & 157.6 \\
$2 / 3$ & $1 / 3$ & 393.6 & 513.6 & 120 & 435 & 600.9 & 165.9 \\
\hline
\end{tabular}

As seen form table 2 4, along with filling rates variation, the change trend of the band gap is not obvious, no matter the single-change or doublechanges TM quasi-periodic phononic crystal.

The change trend of band-gaps frequency is similar, in the single-change or double-changes TM quasi-periodic phononic crystal. With the material 1 's $(\mathrm{Pb})$ filling rate increases or the material 2's (Silicone Rubber) decreases, the start frequency and stop frequency of the first 3 band gap decrease first and then increases, no matter the single-change or doublechanges TM quasi-periodic phononic crystal. Moreover, when the material 1 's ( $\mathrm{Pb}$ ) filling rate is equal to the material 2's (Silicone Rubber), the start frequency and stop frequency of the first 3 band gaps will approach to the minimum value. It is shown that, in order to obtain a band gap whose frequency is low enough, the rigid materials' filling rate should be equal to the elastic materials'.

However, the change trend of band-gaps bandwidth is not the same. For the single-change TM quasi-periodic phononic crystal, the bandwidth of the first band gap increases, the bandwidth of the second and third band gaps increase slowly at first, but then increase rapidly with the material 1 's (Pb) filling rate increase or the material 2's (Silicone Rubber) decrease. For the double-changes TM quasi-periodic phononic crystals, the bandwidth of the second band gap increases, and the bandwidth of the first and third band gaps decrease slowly and then increase, with the material 1's (Pb) filling rate increase or the material 2's (Silicone Rubber) decreases. It is shown that, in order to obtain a band gap whose bandwidth is wide enough, the rigid materials' filling rate should be increased as much as possible.

\subsection{Effects of Layer-Thickness Rate on the Band Gaps}

The layer-thickness rate of material $1(\mathrm{~Pb})$ can be defined as $\mathrm{R} 1=\left(w_{1}+\Delta w_{1}\right) /\left(w_{1}-\Delta w_{1}\right)$, similarly, the layer-thickness rate of material 2 (silicone rubber) as $\mathrm{R} 2=\left(w_{2}+\Delta w_{2}\right) /\left(w_{2}-\Delta w_{2}\right)$. And, to some extent, the value of R1 and R2 represents the quasi periodicity of quasi-periodic phononic crystal with the layer thickness arranged by TM sequence. Obviously, if $\mathrm{R} 1=\mathrm{R} 2=1$, the quasi-periodic phononic crystals with layer 
thickness arranged by the TM sequence will degenerate to periodic structure. The larger the value of $|1-\mathrm{R} 1|$ and $|1-\mathrm{R} 2|$, the greater disparities of specialty between the quasi-periodic phononic crystals and the periodic structures, and the quasi periodicity will be more and more obvious.

Under the condition that filling rate, total thickness and R2 keep constant, the effects of R1 on the band gaps are studied, through the dispersion relation of the quasi-periodic phononic crystal with layer thickness arranged by the TM sequence. Results are shown in table $5 \sim 7$ below.

Table 5: The Effects of R1 on the First Band Gap

\begin{tabular}{c|ccc|ccc}
\hline R1 & Single-Change TM Quasi-Periodic Phononic Crystals & \multicolumn{4}{|c}{ Double-Changes TM Quasi-Periodic Phononic } \\
Crystals & \\
& $\begin{array}{c}\text { Start Frequency } \\
(\mathrm{Hz})\end{array}$ & $\begin{array}{c}\text { Stop Frequency } \\
(\mathrm{Hz})\end{array}$ & $\begin{array}{c}\text { Bandwidth } \\
(\mathrm{Hz})\end{array}$ & $\begin{array}{c}\text { Start Frequency } \\
\text { (Hz) }\end{array}$ & $\begin{array}{c}\text { Stop Frequency } \\
(\mathrm{Hz})\end{array}$ & $\begin{array}{c}\text { Bandwidth } \\
\text { (Hz) }\end{array}$ \\
\hline 2 & 221 & 266.7 & 45.7 & 212.5 & 249.9 & 37.4 \\
3 & 211.6 & 272.2 & 60.6 & 209.8 & 242.4 & 32.6 \\
4 & 206.4 & 271.6 & 65.2 & 207.5 & 238.3 & 30.8 \\
5 & 203.2 & 269.9 & 66.7 & 205.7 & 235.7 & 30 \\
6 & 200.9 & 268.4 & 67.5 & 204.3 & 234.1 & 29.8 \\
7 & 199.3 & 267 & 67.7 & 203.3 & 232.5 & 29.2 \\
8 & 198 & 265.9 & 67.9 & 202.4 & 231.5 & 29.1 \\
9 & 197.1 & 265 & 67.9 & 201.8 & 230.6 & 28.8 \\
\hline
\end{tabular}

Table 6: The Effects of R1 on the Second Band Gap

\begin{tabular}{l|ccc|ccc}
\hline \multirow{2}{*}{ R1 } & Single-Change TM Quasi-Periodic Phononic Crystals & \multicolumn{4}{|c}{ Double-Changes TM Quasi-Periodic Phononic } \\
Crystals
\end{tabular}

Table 7: The Effects of R1 on the Third Band Gap

\begin{tabular}{|c|c|c|c|c|c|c|}
\hline \multirow{2}{*}{ R1 } & \multicolumn{3}{|c|}{ Single-Change TM Quasi-Periodic Phononic Crystals } & \multicolumn{3}{|c|}{$\begin{array}{l}\text { Double-Changes TM Quasi-Periodic Phononic } \\
\text { Crystals }\end{array}$} \\
\hline & $\begin{array}{c}\text { Start Frequency } \\
(\mathrm{Hz})\end{array}$ & $\begin{array}{c}\text { Stop Frequency } \\
(\mathrm{Hz})\end{array}$ & $\begin{array}{c}\text { Bandwidth } \\
(\mathrm{Hz})\end{array}$ & $\begin{array}{l}\text { Start Frequency } \\
(\mathrm{Hz})\end{array}$ & $\begin{array}{l}\text { Stop Frequency } \\
(\mathrm{Hz})\end{array}$ & $\begin{array}{c}\text { Bandwidth } \\
(\mathrm{Hz})\end{array}$ \\
\hline 2 & 346.8 & 421.2 & 74.4 & 375.9 & 509.8 & 133.9 \\
\hline 3 & 356.9 & 465.5 & 108.6 & 399.5 & 550.2 & 150.7 \\
\hline 4 & 375 & 508.1 & 133.1 & 424.1 & 590.5 & 166.4 \\
\hline 5 & 392.9 & 538.3 & 145.4 & 447.2 & 627 & 179.8 \\
\hline 6 & 411.5 & 567.2 & 155.7 & 468.7 & 657.9 & 189.2 \\
\hline 7 & 427.6 & 594 & 166.4 & 488.1 & 686.5 & 198.4 \\
\hline 8 & 442.1 & 618.1 & 176 & 506 & 712 & 206 \\
\hline 9 & 455.5 & 640.2 & 184.7 & 522.3 & 734.9 & 212.6 \\
\hline
\end{tabular}

As seen form table $5 \sim 7$, for the single-change TM quasi-periodic phononic crystal, with the increase of R1, the first 3 band gaps' bandwidth increase, the start frequency of the first and second band gaps move to higher area, and both of the third band gap's start frequency and stop frequency move to higher area. For the double-changes TM quasi-periodic phononic crystal, with the increase of R1, the bandwidth of the first band gap narrows, and the bandwidth of the second and third band gaps become wider; the start frequency and stop frequency of the first band gap, and the start frequency of the second band gap decrease; the stop frequency of the second band gap, and the start frequency and stop frequency of the third band gap increase.

Under the condition that filling rate, total thickness and R1 keep constant, the effects of R2 on the band gaps is studied. Results are shown in table $8 \sim 10$ below.

Table 8: The Effects of R2 on the First Band Gap

\begin{tabular}{l|ccc|ccc}
\hline R2 & Single-Change TM Quasi-Periodic Phononic Crystals & \multicolumn{4}{|c}{ Double-Changes TM Quasi-Periodic Phononic } \\
Crystals & \\
& $\begin{array}{c}\text { Start Frequency } \\
(\mathrm{Hz})\end{array}$ & $\begin{array}{c}\text { Stop Frequency } \\
(\mathrm{Hz})\end{array}$ & $\begin{array}{c}\text { Bandwidth } \\
(\mathrm{Hz})\end{array}$ & $\begin{array}{c}\text { Start Frequency } \\
(\mathrm{Hz})\end{array}$ & $\begin{array}{c}\text { Stop Frequency } \\
(\mathrm{Hz})\end{array}$ & $\begin{array}{c}\text { Bandwidth } \\
(\mathrm{Hz})\end{array}$ \\
\hline 2 & 220.7 & 266.6 & 45.9 & 212.6 & 250.4 & 37.8 \\
3 & 211.4 & 270.3 & 58.9 & 209.8 & 242.4 & 32.6 \\
4 & 206.3 & 268.4 & 62.1 & 207.5 & 238 & 30.5 \\
5 & 203.2 & 266.2 & 63 & 205.7 & 235.2 & 29.5 \\
6 & 201 & 264 & 63.1 & 204.4 & 233.2 & 28.8 \\
7 & 199.4 & 262.4 & 63 & 203.3 & 231.7 & 28.4 \\
8 & 198.2 & 261.1 & 62.9 & 202.6 & 230.6 & 28 \\
9 & 197.2 & 260 & 62.8 & 201.9 & 229.7 & 27.8 \\
\hline
\end{tabular}

Table 9: The Effects of R2 on the Second Band Gap

\begin{tabular}{l|ccc|ccc}
\hline R2 & Single-Change TM Quasi-Periodic Phononic Crystals & \multicolumn{4}{|c}{ Double-Changes TM Quasi-Periodic Phononic } \\
Crystals & \\
& $\begin{array}{c}\text { Start Frequency } \\
(\mathrm{Hz})\end{array}$ & $\begin{array}{c}\text { Stop Frequency } \\
(\mathrm{Hz})\end{array}$ & $\begin{array}{c}\text { Bandwidth } \\
(\mathrm{Hz})\end{array}$ & $\begin{array}{c}\text { Start Frequency } \\
(\mathrm{Hz})\end{array}$ & $\begin{array}{c}\text { Stop Frequency } \\
(\mathrm{Hz})\end{array}$ & $\begin{array}{c}\text { Bandwidth } \\
(\mathrm{Hz})\end{array}$ \\
\hline 2 & 297.8 & 330.3 & 32.5 & 266.1 & 366.8 & 100.7 \\
3 & 286.1 & 359.4 & 73.3 & 256.1 & 398.5 & 142.4 \\
4 & 279 & 399.9 & 120.9 & 250.5 & 429.5 & 179 \\
5 & 274.4 & 417.2 & 142.8 & 246.9 & 459.5 & 212.6 \\
6 & 271.2 & 444 & 172.8 & 244.4 & 488.2 & 243.8 \\
7 & 268.7 & 469.5 & 200.8 & 242.6 & 515.6 & 273 \\
8 & 266.9 & 493.8 & 226.9 & 241.2 & 541.6 & 300.4 \\
9 & 265.5 & 516.5 & 251 & 240 & 566.4 & 326.4 \\
\hline
\end{tabular}

Table 10: The Effects of R2 on the Third Band Gap

\begin{tabular}{|c|c|c|c|c|c|c|}
\hline \multirow{2}{*}{ R2 } & \multicolumn{3}{|c|}{ Single-Change TM Quasi-Periodic Phononic Crystals } & \multicolumn{3}{|c|}{$\begin{array}{l}\text { Double-Changes TM Quasi-Periodic Phononic } \\
\text { Crystals }\end{array}$} \\
\hline & $\begin{array}{c}\text { Start Frequency } \\
(\mathrm{Hz})\end{array}$ & $\begin{array}{c}\text { Stop Frequency } \\
(\mathrm{Hz})\end{array}$ & $\begin{array}{c}\text { Bandwidth } \\
(\mathrm{Hz})\end{array}$ & $\begin{array}{c}\text { Start Frequency } \\
(\mathrm{Hz})\end{array}$ & $\begin{array}{l}\text { Stop Frequency } \\
(\mathrm{Hz})\end{array}$ & $\begin{array}{c}\text { Bandwidth } \\
(\mathrm{Hz})\end{array}$ \\
\hline 2 & 348.2 & 425.4 & 77.2 & 371.2 & 500.7 & 129.5 \\
\hline 3 & 365.1 & 476 & 110.9 & 399.5 & 550.2 & 150.7 \\
\hline 4 & 391.2 & 522.3 & 131.1 & 430 & 599.3 & 169.3 \\
\hline 5 & 418.4 & 564.9 & 146.5 & 496 & 649.2 & 153.2 \\
\hline 6 & 445 & 604 & 159 & 488.5 & 688.6 & 200.1 \\
\hline 7 & 470.1 & 641.8 & 171.7 & 515.8 & 726.2 & 210.4 \\
\hline 8 & 494.2 & 676.6 & 182.4 & 541.8 & 763 & 221.2 \\
\hline 9 & 517 & 709.6 & 192.6 & 566.5 & 797.5 & 231 \\
\hline
\end{tabular}

As seen form table 8 10, along with R2 variation, the band gaps of the single-change and double-changes TM quasi-periodic phononic crystals have roughly same varying trend. 
With the increase of $\mathrm{R} 2$, the start frequencies of the second band gaps decrease, the stop frequencies of the second band gaps increase, in the single-change and double-changes TM quasi-periodic phononic crystals. Because the stop frequencies of the third band gaps increase much faster than their start frequencies, the bandwidth of the third band gaps increase.

However, the major band-gaps difference between the single-change and double-changes TM quasi-periodic phononic crystals is shown in their first band gap. With the increase of R2, the start frequency of the first band gap of the single-change TM quasi-periodic phononic crystal decreases, and its stop frequency increases first and then decreases, thus its bandwidth increases first and then narrows. On the other hand, the start frequency and stop frequency of the first band gap of the double-changes TM quasiperiodic phononic crystal decrease, and its bandwidth becomes narrow, with the increase of R2.

It can be concluded that adjusting the layer-thickness rate can adjust the band gap distribution of the layer thickness quasi-periodic structure phononic crystal, and increasing the material layer-thickness rate can make the first band gap start frequency decreased, especially when increasing the ratio of the rigid materials' layer thickness, not only the first band gap of the TM quasi - periodic structure phononic crystal, but also the first band gap bandwidth can be increased, which greatly improves the practical application value of the quasi-periodic phononic crystal vibration isolator.

\section{CONCLUSION}

In this paper, the band structure of one dimensional quasi-periodic phononic crystals with layer thickness arranged by TM sequence is calculated, using the transfer matrix method combined with the supercell method, and the effects of filling rate and layer-thickness rate on band gaps are discussed. The conclusions are as follows:

a) Compared with the periodic structure, the first band gap frequency of one dimensional quasi-periodic phononic crystals with layer thickness arranged by TM sequence is significantly reduced, which is beneficial to their practical application.

b) The desired low-frequency band gap can be obtained by adjusting the materials' filling rate. Keeping the rigid materials' filling rate equal to the elastic materials', the start frequency and stop frequency of the band gap will move to lower area.

c) Changing layer-thickness rate is a useful way to redistribute the band gap of the quasi-periodic phononic crystals with layer thickness arranged by TM sequence. Especially when increase the rigid materials' layer-thickness rate, not only the first band gap of the single-change TM quasi-periodic phononic crystal can be reduced, but also its first band gap bandwidth can be increased, which greatly improves the practical application value of the single-change TM quasi-periodic phononic crystal vibration isolator.

\section{ACKNOWLEDGMENTS}

This work is founded by the National Science Foundation of China under grant numbers 61503354 . The support is gratefully acknowledged.

\section{REFERENCES}

[1] Xi-seng, W., Ji-hong, W., Dian-long, Y. 2009. Phononic Crystals. Beijing: National Defense Industry Press.

[2] Yan, Z., Lin, H., Lin-hua, J. 2015. Phononic Crystal Calculation Method and Band Gap Properties. Beijing: Science Press.

[3] Jiu-hui, W. 2014. The Vibration and Noise of the Frontier Theory and Application. Xi'an: Xi'an Jiaotong University Press.

[4] Hong-xing, D. 2014. Study on the propagation characteristics of laser-induced Lamb waves in (quasi-)periodic structure plates. Nanjing: Nanjing University of Science and Technology.

[5] Yong-jun, C., Chun-hong, D., Pei-qin, Z. 2008. Band gap characteristics of one-dimensional phononic crystal with quasi-periodical structure. Journal of inner mongolia normal university (natura science edition), 37, 53-57.

[6] Gao, J., Chun-cheng, J. 2007. Propagation of lamb waves in onedimensional quasiperiodic composite thin plates: a split of phonon band gap. Applied physics letters, 90, 111908-111908-3.

[7] Li-feng, Y., Ya-fei, W., Ying, Z. 2012. The transmission properties in one-dimensional piezoelectric fibonacci-class quasi-periodical phononic crystals. Acta physica sinica, 61, 425-430.

[8] Xu-yang, X., Run-pin, C., Ji-hong, X. 2014. Propagation of elastic wave in a symmetrical quasi-periodic phononic crystal composed of solid/liquid. Journal of Vibration and Shock, 33, 122-126.

[9] Lei, L., Yong-shou, L., Jian-ting, R. 2012. Band gap characteristics of Thue-Morse (TM) one dimensional rod phononic crystal with quasiperiodic structure. Journal of northwestern polytechnical university, 30, 98-103.

[10] Mai-ling, C. 2010. Thermal conduction properties of one dimensional quasi periodic system. Changsha: Central south university.

[11] Zheng-dong, W., Bao-ren, L., Jing-min, D. 2016. Research on the longitudinal vibration band gaps of isolator applied to ship hydraulic pipesupport based on the theory of phononic crystals. Journal of mechanical engineering, 52, 97-104.

[12] Peng-shan, Q., Jun, D., Jiu-long, J. 2016. Band gap characteristics of the layered phononic crystal with double-cycle structure. Technical acoustics, 35, 25-29.

[13] A-li, C., Feng-dan, G., Yue-sheng, W. 2015. Study on the bandgaps of the lame wave in one-dimensional quasi periodic phononic crystal plates. Journal of functional materials, 46, 19046-19051. 